\title{
A New Dual-bitstream Video Streaming System with VCR Functionalities Using SP/SI-frames
}

\author{
Yui-Lam Chan • Tak-Piu Ip • Ki-Kit Lai • \\ Chang-Hong Fu $\cdot$ Wan-Chi Siu
}

Received: 30 December 2009/Revised: 30 December 2009/Accepted: 4 March 2010/Published online: 24 March 2010

(C) Springer Science+Business Media, LLC 2010

\begin{abstract}
With the proliferation of digital video and the popularity of video streaming applications, it is highly desirable to find and access video segments of interest by searching through the content of video at a speed that is faster than a normal playback. The key functions that enable quick browsing of video are fast-forward and fastreverse playbacks. However, motion-compensated prediction adopted in the current video coding standards drastically complicates these operations. One approach to implement the fast-forward/reverse playback is to store an additional reverse-encoded bitstream in the server. Once the client requests a fast-forward/reverse operation, the server can select an appropriate frame for the client from either the forward-encoded bitstream or the reverse-encoded bitstream by considering the cost of network bandwidth and decoder complexity. Unfortunately, these two bitstreams are encoded separately. The frame in one bitstream may not be exactly identical to the frame in another bitstream. If one of these frames is then used as the reference for the requested frame, which is in another bitstream, it induces mismatch errors. In this paper, a novel H.264 dualbitstream system aiming at providing the fast-forward/ reverse playback based on SP/SI-frames is proposed. The proposed system can completely eliminate mismatch errors when the frame in the reverse-encoded bitstream replaces the frame in the forward-encoded bitstream and vice versa.
\end{abstract}

Y.-L. Chan $(\bowtie) \cdot$ T.-P. Ip • K.-K. Lai • C.-H. Fu • W.-C. Siu

Centre for Signal Processing,

Department of Electronic and Information Engineering,

The Hong Kong Polytechnic University,

Hung Hom,

Kowloon, Hong Kong

e-mail: enylchan@polyu.edu.hk
Experimental results confirm that the proposed system is effective in eliminating mismatch errors so as to enhance the performance of the dual-bitstream system.

Keywords Digital video cassette recording (VCR) . MPEG/H.264 · Streaming video · Dual-bitstream system

\section{Introduction}

Streaming video over the Internet becomes popular in recent years, mainly due to the continued advance of video compression and broadband networking technologies $[1,2]$. These streaming applications such as video-on-demand allow users to ubiquitously access and retrieve various videos over networks using software players [3, 4] or digital set-top box devices $[5,6]$, which are currently ready to take place of obsolete analog-based devices and systems such as analog TV and Video Cassette Recorders (VCRs). Nowadays, the digital video coding standards such as MPEG-1/2/4 [7-9] and H.26x series [10, 11] were developed primarily for achieving high compression ratios by using motion-compensated prediction [12-14]. In order to complete the transition to digital video from its current analog state, it is highly desirable that users can browse video contents in a fast and user-friendly way [15-18]. Fast-forward and fast-reverse playbacks are two popular video browsing functions provided in many analog and digital video players. However, motion-compensated prediction is mainly designed and best suited for a normal playback in which frames are decoded and played back in a pre-determined frame order. The frame dependency severely complicates the fast-forward/reverse operations since a predicted frame can only be decoded when all its reference frames have already been decoded. 
Some works on the implementation of fast playbacks for video streaming were introduced in [15-22]. In [19], these operations are implemented at the client side by using prefetched video frames. However, this approach requires a huge buffer in the client machine, which is not desirable. Although the frame-skipping video transcoding methods proposed in $[20,21]$ can be used to support fast forward/ reverse playbacks, they introduce much computation in the server and will cause drift due to the transcoding process. Omoigui et al. [22] suggested a number of client-server timecompression solutions for a fast-forward playback and video browsing. By using time-compression, the server stores multiple pre-encoded video bitstreams with various temporal resolutions and transmits a video bitstream with appropriate temporal resolution according to the speed-up factor required by users. This approach does not induce excessive buffers in the client but the speed-up granularity is limited by the number of pre-stored video bitstreams. In [15-17], some schemes proposed to store a forward-encoded bitstream and a reverse-encoded bitstream in the server. The idea is to switch frames between the forward-encoded bitstream and the reverse-encoded bitstream based on a frame-selection scheme, which is used to minimize the number of transmitted frames over the network for any speed-up factors. This dual-bitstream system can alleviate the decoder complexity while maintaining the low network bandwidth requirement in fast playbacks. However, this approach would cause a mismatch problem because a P-frame in the reverseencoded bitstream may have to be approximated by an I-frame in the forward-encoded bitstream and vice versa.

In this paper, we utilize the concept of SP/SI-frame coding [23, 24] and provide an efficient solution for performing various fast playbacks in the dual-bitstream streaming system to eliminate the problem arising from the mismatch between the forward-encoded and reverseencoded bitstreams. Our system is designed specifically for H.264 and can be adapted to any other coding standards that support SP/SI-frames. It is also a new application of $\mathrm{SP} / \mathrm{SI}$-frames. The organization of this paper is as follows. Section 2 of this paper presents an in-depth study of the problems on the dual-bitstream system. In Section 3, a brief introduction of SP/SI-frame coding for H.264 is given and the proposed system is discussed in detail, where we describe how to adopt SP/SI-frames in the dual-bitstream system. We also illustrate how to modify the encoding processes of SP/SI-frames such that they work well with the newly modified dual-bitstream system. Experimental results are presented in Section 4. Finally, some concluding remarks are provided in Section 5.

\section{Dual-bitstream MPEG Video Streaming System}

To find a way to deal with the problem in different VCR trick modes of digital video, a dual-bitstream system was proposed in [15] which adds a reverse-encoded bitstream (RB) in the server in addition to the traditional forwardencoded bitstream (FB). The generation of the RB is simply encoding the video in reverse order. Figure 1 shows an illustrative example of the $\mathrm{FB}$ and the $\mathrm{RB}$ in which the video is coded in I/ P-frames with a GOP size of 14 frames and I-frames in the RB is interleaved between I-frames in the FB. Note that B-frames are not used as references for later frames. It means they are not involved in decoding other frames. For simplicity but without loss of generality, we focus our discussions on the case that the video stream contains I/P-frames only. Once the server receives the VCR command or the requested frame number from the client, the server employs a frame-selection scheme to determine which frames in either the FB or the RB to be transmitted by minimizing the cost of decoding the next requested frame. In general, a larger number of frames to be sent induces much heavier network traffic and higher decoding complexity. For this reason, the cost can be approximated to the number of frames to be sent over the network [15]. The frame-selection scheme actually measures the distances from the next requested frame to the current displayed frame $\left(d_{C}\right)$, the nearest I-frame in the FB $\left(d_{F B}\right)$ and the nearest I-frame in the RB $\left(d_{R B}\right)$. It then picks the frame with the minimum distance as the reference frame to the next requested frame to initiate the decoding. Since this selected reference frame has a shorter distance to the next requested

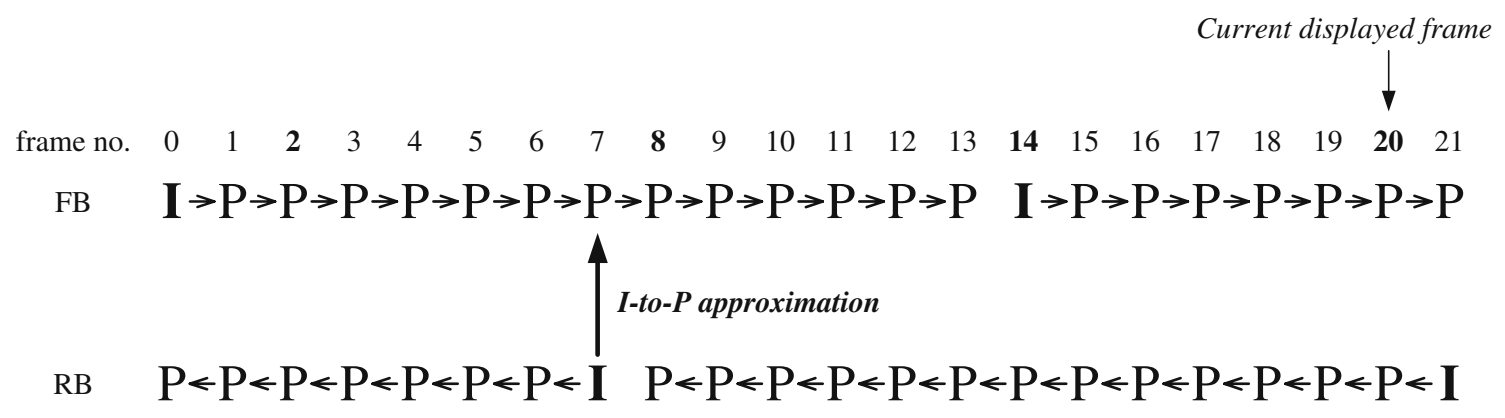

Figure 1 Fast-reverse operation with the speed-up ratio of 6 in the dual-bitstream video streaming system. 
frame, a smaller number of frames is required to be sent. The frame-selection scheme may switch from the FB to the $\mathrm{RB}$ and vice versa according to the current play-direction, the requested mode and the distances $d_{C}, d_{F B}$ and $d_{R B}$. In other words, it will determine which bitstream to be selected next and its decoding direction. To illustrate the scheme, let us recall the structure of dual bitstreams in Fig. 1 again. Assume that the previous mode was in the normal forward playback. At frame 20, fast-reverse playback with a speed-up factor of 6 is requested. In this case, frames 14,8 , and 2 should be displayed. If the requested frame is an I-frame in one of two bitstreams, the frame can be decoded by itself. Thus, in the above example, frame 14 will be decoded from the FB directly since it is an I-frame. Then, the next frame to be decoded is frame 8 . Since the requested frame is a P-frame in both bitstreams, the current displayed frame, or the nearest I-frame either in the $\mathrm{FB}$ or the $\mathrm{RB}$ is firstly selected to initiate the decoding of the requested frames. In this example, frame 8 will be decoded from frame 7 of the RB (an I-frame) since $d_{R B}$ has the smallest value among all distances. It implies the nearest I-frame of the RB (frame 7) is the closest reference to frame 8. Note that frame 7 of the RB (an I-frame) is used as an approximation of frame 7 of the FB (a P-frame) to reconstruct frame 8 of the FB, as depicted in Fig. 1. This Ito-P approximation will cause the drift problem due to the mismatch between the reference frames. Furthermore, the drift will not only be confined to the frame at the switching location, but will further propagate in time if it is used as reference for other frames. This is the case when the current fast-reverse mode is switched back to the normal playback at frame 8 . In this situation, frame 9 will be decoded by using the drifted frame 8 . This drift continues until the next I-frame in the FB. The quality degradation in this situation is depicted in Fig. 2 where the test video used for simulation is "Foreman" sequence. The "Foreman" sequence is encoded at $330 \mathrm{~Kb} / \mathrm{s}$ with a frame-rate of $30 \mathrm{fps}$ and the GOP length is 14 with an I-P structure. The starting point of the fast-reverse operation with a speed-up factor of 6 is at frame 20 and the normal-play operation is then requested at frame 8 . This figure shows that the drift caused by the bitstream switching can be as large as $1.3 \mathrm{~dB}$ and will last until the next I-frame in the FB. Although the drift problem can be compensated by adding two driftcompensated bitstreams [15], it increases the storage requirements of the server.

\section{Proposed Dual-bitstream Video System Based on SP/SI-frames}

In the paper, we suggest an efficient solution for the drift problem by modifying the original structure of the dual

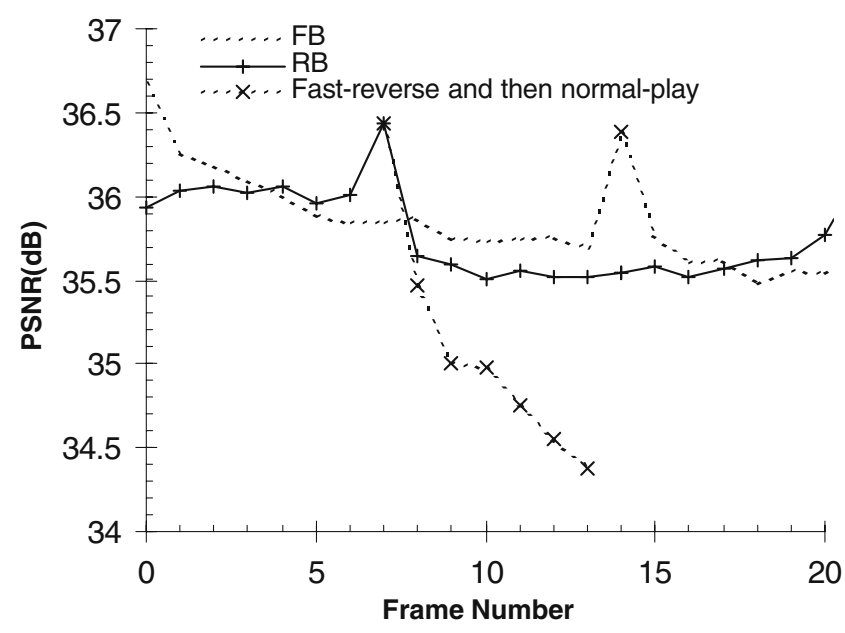

Figure 2 Quality degradation in the situation when the fast-reverse operation with a speed-up factor of 6 is requested at frame 20 and then the normal playback is issued at frame 8 .

bitstreams with the help of SP/SI-frame coding. The new structure provides seamlessly approximation between frames in the FB and the RB for any VCR operation. SP/SI-frames are new picture types supported by H.264 [23, 24]. The traditional usage of SP/SI-frames is for drift-free switching between compressed bitstreams of different bit rates to accommodate the channel bandwidth variation [23-26]. Figure 3 shows an example in which a video is coded into two bitstreams (bitstream A and bitstream B) with different bit rates. Within each encoded bitstream, two SP-frames$S P_{A, n}$ and $S P_{B, n}$, as shown in Fig. 3, are placed at frame $n$ at which switching from one bitstream to another will be allowed. These SP-frames are referred to as primary SPframes. Besides, for each primary SP-frame, a corresponding secondary SP-frame $\left(S P_{A B, n}\right.$ in Fig. 3$)$ is generated, which has the same reconstructed values as the primary SP-frame. Such a secondary SP-frame is sent only during bitstream switching. For normal transmission, either bitstream A or bitstream $B$ is sent to the user depending on the current available bandwidth. When there is a need to switch from bitstream A to bitstream B at frame $n, S P_{A B, n}$ instead of $S P_{B, n}$ is transmitted. Note that, $S P_{A B, n}$ and $S P_{B, n}$ use different reference frames, $S P_{A, n-1}$ and $S P_{B, n-1}$ respectively, for prediction. After decoding $S P_{A B, n}$, the decoder can obtain exactly the same reconstructed values as normally $S P_{B, n}$ decoded at frame $n$, therefore it can continually decode bitstream B at frame $n+1$ seamlessly. The way to encode $S P_{A B, n}$ ensures that an identical reconstruction as that of $S P_{B, n}$ can be obtained even $S P_{A B, n}$ and $S P_{B, n}$ have different reference frames. Readers interested in learning how such property is achieved are encouraged to read the references $[23,24]$. Similarly, an SI-frame can also be used in the bitstream-switching scenario. The only difference is that its prediction is formed using the intra-prediction modes from previously decoded samples of the reconstructed frame. The 


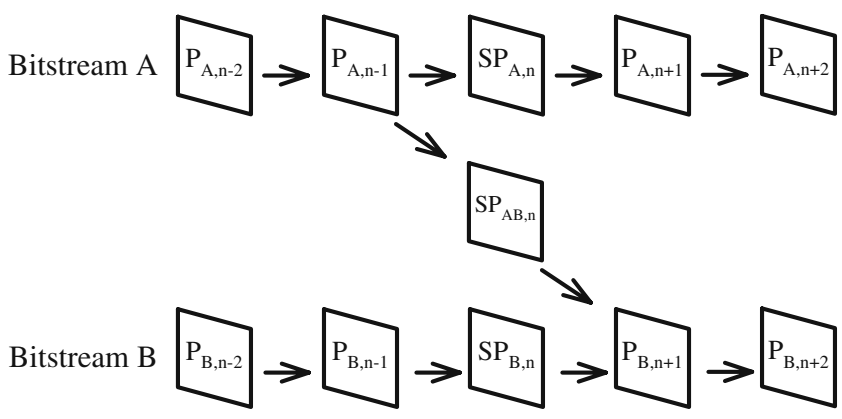

Figure 3 Bitstream switching using SP pictures.

SI-frame may be used when the two bitstreams are completely different. In this case, it will not be efficient to use motion-compensated prediction because there is no correlation between these two sequences.

This property motivates us to adopt SP/SI-frames in the structure of dual bitstreams to prevent reference mismatch due to the I-to-P approximation during the fast playback operations. In the following, we provide a detailed description and formulation of the proposed dual-bitstream system.

\subsection{Dual-bitstream Video System with SP/SI-frames}

As mentioned in Section 2, the conventional dual-bitstream system suffers the drift problem due to the I-to-P approximation during fast playbacks. In our system, we propose to adopt the SP/SI-frame pairs at the switching points which are the points when a frame of one bitstream may be used to replace a frame of the other bitstream. This arrangement ensures that the I-to-P approximation will not happen at switching points for any fast-forward/reverse operation. Consequently, the new dual-bitstream system will not cause any mismatch at the decoder side.

The idea of utilizing SP/SI-frame coding for the purpose of the seamless approximation in VCR operations is introduced in Fig. 4. In the original structure of the dual bitstreams, I-frames represent the points of access to decode the sequence from any arbitrary position. In order to avoid the problem of reference mismatch, instead of using I-frames in the original dual bitstreams, SI-frames are adopted at the switching points. An SI-frame can be placed either in the FB or $\mathrm{RB}$ which is referred to as a forward-encoded SI-frame
$\left(\mathrm{SI}_{\mathrm{FB}}\right)$ or a reverse-encoded SI-frame $\left(\mathrm{SI}_{\mathrm{RB}}\right)$ respectively. For each $\mathrm{SI}_{\mathrm{FB}}$, there is a corresponding reverse-encoded SPframe $\left(\mathrm{SP}_{\mathrm{RB}}\right)$ and these two frames form an $\mathrm{SI}_{\mathrm{FB}} / \mathrm{SP}_{\mathrm{RB}}$ pair. Similarly, there is a corresponding forward-encoded SPframe $\left(\mathrm{SP}_{\mathrm{FB}}\right)$ for each $\mathrm{SI}_{\mathrm{RB}}$ and they form an $\mathrm{SP}_{\mathrm{FB}} / \mathrm{SI}_{\mathrm{RB}}$ pair. Identical frame reconstruction can be allowed at these $\mathrm{SI}_{\mathrm{FB}} /$ $\mathrm{SP}_{\mathrm{RB}}$ and $\mathrm{SP}_{\mathrm{FB}} / \mathrm{SI}_{\mathrm{RB}}$ pairs when an SI-frame of one bitstream is used to replace an SP-frame of the other bitstream. This facilitates the correctly predicted frame to be used if the replacement of SP with SI is needed in any VCR operation and hence no drift will occur. To illustrate the proposed system, we use the example in Fig. 1 again in which the current VCR is in the fast-reverse mode and this operation requires to display frames 14,8 , etc. After decoding and displaying frame 8 , then a normal-play request is launched. Frame 8 will be decoded from frame 7 of the $\mathrm{RB}$, which is an $\mathrm{SI}_{\mathrm{RB}}$. After decoding this $\mathrm{SI}_{\mathrm{RB}}$, the decoder can obtain exactly the identical reconstruction as normally decoding $\mathrm{SP}_{\mathrm{FB}}$ at frame 7 in the $\mathrm{FB}$, therefore it can decode frame 8 in the FB without any mismatch error and continue to decode the subsequent frames seamlessly when the normal playback is requested at frame 8 . Hence, the proposed dual-bitstream system does not suffer the drift problem because no approximation between the SI-frame and the SP-frame on the dual bitstreams has been made.

\subsection{Encoding and Decoding Arrangement of $\mathrm{SP}_{\mathrm{FB}} / \mathrm{SI}_{\mathrm{RB}}$ and $\mathrm{SI}_{\mathrm{FB}} / \mathrm{SP}_{\mathrm{RB}}$ Pairs}

To ensure the identical reconstruction at switching pairs, this section provides a detailed description of how to encode the $\mathrm{SP}_{\mathrm{FB}} / \mathrm{SI}_{\mathrm{RB}}$ and $\mathrm{SI}_{\mathrm{FB}} / \mathrm{SP}_{\mathrm{RB}}$ pairs. Figure 5(a) shows the encoding processes of $\mathrm{SP}_{\mathrm{FB}}$ at frame $n\left(S P_{F B, n}\right)$ and its corresponding $\mathrm{SI}_{\mathrm{RB}}\left(S I_{R B, n}\right)$. Similar to SP-frame encoding [23, 24], some additional quantization processes are required. For the sake of convenience, we use the superscript $r$ to denote the reconstructed frame or the reconstructed prediction error, and the capital letter with superscript $Q_{p}$ or $Q_{s}$ to represent the coefficients in quantized transform domain with the quantization level $Q_{p}$ or $Q_{s}$ respectively for the rest of this paper.

A predicted frame is formed by motion-compensated prediction using the original frame $n\left(O_{F B, n}\right)$ in the FB and

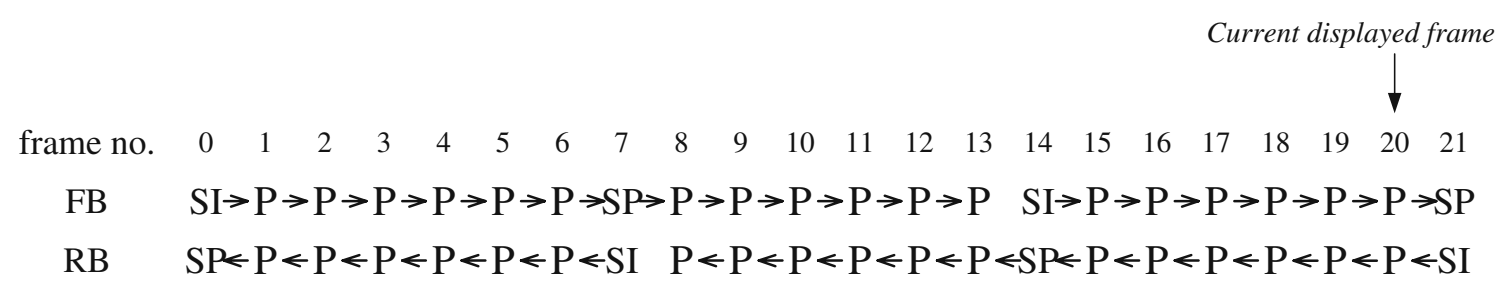

Figure 4 Structure of the proposed dual bitstreams with SP/SI-frame pairs. 
Figure 5 Encoding and decoding of an $\mathrm{SP}_{\mathrm{FB}} / \mathrm{SI}_{\mathrm{RB}}$ pair. (a) $\mathrm{SP}_{\mathrm{FB}}$ and $\mathrm{SI}_{\mathrm{RB}}$ encoding, (b) $\mathrm{SP}_{\mathrm{FB}}$ decoding, and (c) $\mathrm{SI}_{\mathrm{RB}}$ decoding.

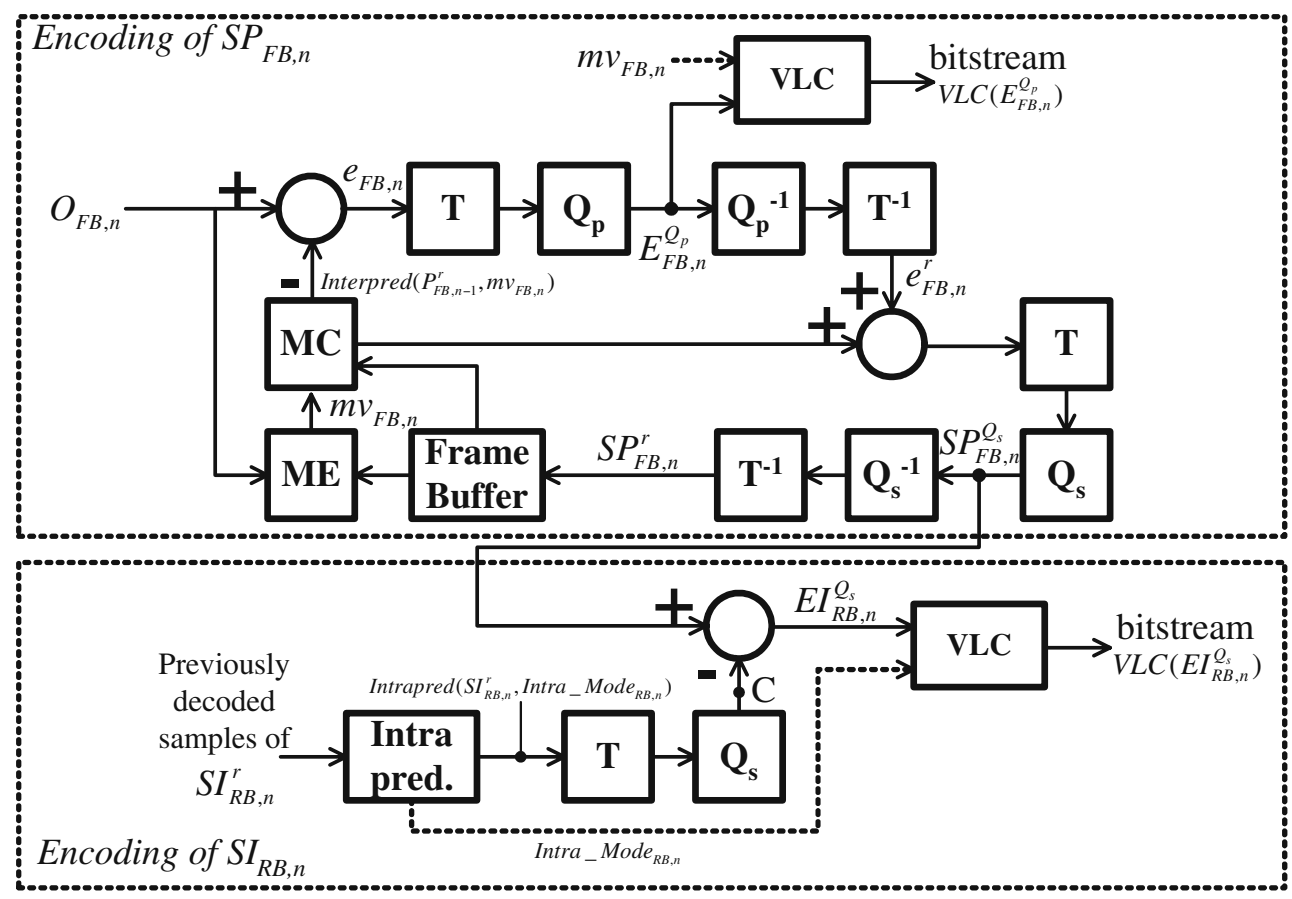

a

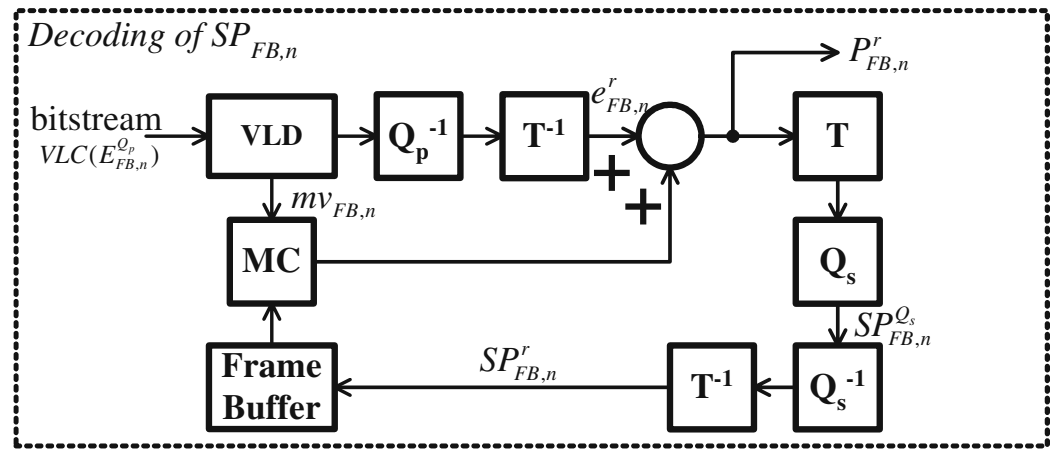

b

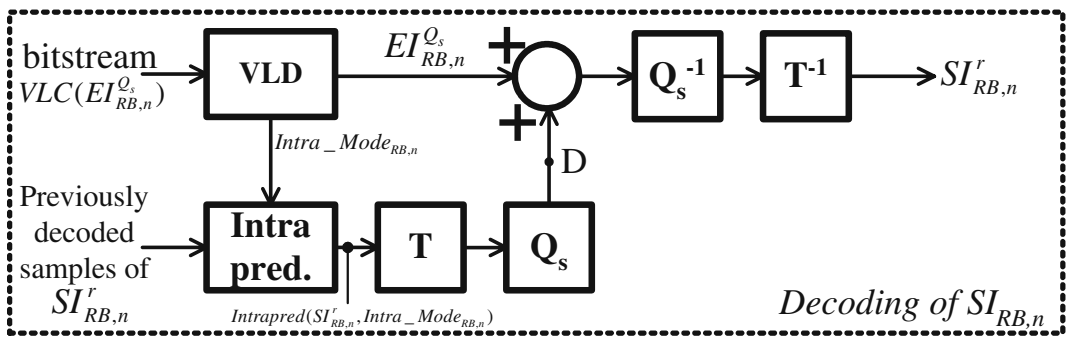

C

the previously reconstructed frame $n-1, P_{F B, n-1}^{r}$, stored in the frame buffer. It can be written as Interpred $\left(P_{F B, n-1}^{r}, m v_{F B, n}\right)$ where Interpred( ) is the motion-compensated prediction operator and $m v_{F B, n}$ represents the set of motion vectors of frame $n$ in FB. The prediction error $e_{F B, n}$ between $O_{F B, n}$ and its prediction is

$e_{F B, n}=O_{F B, n}-$ Interpred $\left(P_{F B, n-1}^{r}, m v_{F B, n}\right)$
Performing the transformation and quantization on $e_{F B, n}$ with $Q_{p}$, we get

$E_{F B, n}^{Q_{p}}=Q_{p}\left(T\left(e_{F B, n}\right)\right)$

$E_{F B, n}^{Q_{p}}$ is then compressed into the bitstream $\operatorname{VLC}\left(E_{F B, n}^{Q_{p}}\right)$ with entropy coding. The above encoding process is exactly the same as normal P-frame encoding. To generate $S P_{F B, n}$, 
an additional quantization process is applied to the P-frame. This additional quantization process ensures that the transform coefficients of the reconstructed frame $S P_{F B, n}^{r}$ can be quantized and de-quantized without loss at the quantization level $Q_{s}$, which is going to be used in the encoding process of $S I_{R B, n}$. Note that the server using the proposed dualbitstream system with SP/SI-frames still stores $\operatorname{VLC}\left(E_{F B, n}^{Q_{p}}\right)$ instead of the bitstream generated by $S P_{F B, n}$. This $E_{F B, n}^{Q_{p}}$ can be used to generate $S P_{F B, n}^{r}$ in the local decoder loop of the SPframe encoder as well as the decoder. In the following, we will describe how to generate $S P_{F B, n}^{r}$ which is used to update the frame buffers in both encoder and decoder to act as a reference for the next P-frame.

Before performing an additional quantization process on the P-frame, the reconstructed prediction error $e_{F B, n}^{r}$ are obtained by applying dequantization with the quantization level $Q_{p}$ and then inverse transformation on $E_{F B, n}^{Q_{p}}$.

$e_{F B, n}^{r}=T^{-1}\left(Q_{p}^{-1}\left(E_{F B, n}^{Q_{p}}\right)\right)$

The reconstructed prediction error $e_{F B, n}^{r}$ is then added to Interpred $\left(P_{F B, n-1}^{r}, m v_{F B, n}\right)$ for computing the reconstructed P-frame $P_{F B, n}^{r}$, which can be written as

$P_{F B, n}^{r}=e_{F B, n}^{r}+\operatorname{Interpred}\left(P_{F B, n-1}^{r}, m v_{F B, n}\right)$

$P_{F B, n}^{r}$ is then transformed again and the transform coefficients of $P_{F B, n}^{r}$ is quantized using the quantization level $Q_{s}$. The quantized transform coefficients of $P_{F B, n}^{r}$ become

$S P_{F B, n}^{Q_{s}}=Q_{s}\left(T\left(P_{F B, n}^{r}\right)\right)$

After performing dequantization with $Q_{s}$ and inverse transformation on $S P_{F B, n}^{Q_{s}}$, we obtain an expression of the reconstructed frame $S P_{F B, n}^{r}$.

$S P_{F B, n}^{r}=T^{-1}\left(Q_{s}^{-1}\left(S P_{F B, n}^{Q_{s}}\right)\right)$

This reconstructed frame $S P_{F B, n}^{r}$, which is stored in the frame buffer of the encoder, acts as a reference frame for the next P-frame. Equations 5 and 6 indicate that the transform coefficients of $S P_{F B, n}^{r}$ can be quantized and dequantized without loss at the quantization level $Q_{s}$. These represent the fundamental ideas of SP/SI-encoding to ensure identical reconstruction of $S I_{R B, n}$ even when different predictions are used.

The decoding process of $S P_{F B, n}^{r}$ is shown in Fig. 5(b) in which $\operatorname{VLC}\left(E_{F B, n}^{Q_{p}}\right)$ is an input bitstream. The decoder actually is the local decoder loop of the encoding process for $S P_{F B, n}$ as shown in Fig. 5(a). Its decoding operation follows Eqs. 3-6. Finally, an identical $S P_{F B, n}^{r}$ to the encoder is reconstructed to update the frame buffer in the decoder which is used for decoding the next P-frame.

In order to avoid the problem of reference mismatch due to the replacement of $S P_{F B, n}^{r}$ with $S I_{R B, n}^{r}$, the reconstructed frame $S I_{R B, n}^{r}$ at frame $n$ in the RB must be identical to $S P_{F B, n}^{r}$ in the FB. To achieve this, the quantized values of transform coefficients in both $S P_{F B, n}^{r}$ and $S I_{R B, n}^{r}$ must be synchronized to the same quantization level $Q_{s}$. By using the same encoder as shown in Fig. 5(a), $S P_{F B, n}^{Q_{s}}$ acts as an input instead of the original video frame in the $R B$, and the quantization operation is processed before calculating the prediction error of $S I_{R B, n}, E I_{R B, n}^{Q_{s}}$, which can be computed as

$$
E I_{R B, n}^{Q_{s}}=S P_{F B, n}^{Q_{s}}-Q_{s}\left(T\left(\text { Intrapred }\left(S I_{R B, n}^{r}, \text { Intra_Mode }_{R B, n}\right)\right)\right)
$$

where Intrapred( ) is the intra-prediction operator and Intra_Mode $e_{R B, n}$ represents Intra prediction modes. For coding $S I_{R B, n}$, the prediction is formed by using the intraprediction modes from previously decoded samples of the reconstructed frame. Again the encoder compresses in the bitstream $\operatorname{VLC}\left(E I_{R B, n}^{Q_{s}}\right)$ with entropy coding. Since the same quantization level $Q_{s}$ are used for $S P_{F B, n}^{Q_{s}}$ and $Q_{s}\left(T\left(\right.\right.$ Intrapred $\left(S I_{R B, n}^{r}\right.$, Intra_Mode $\left.\left.\left._{R B, n}\right)\right)\right)$ in Eq. 7, $E I_{R B, n}^{Q_{s}}$ is also synchronized at $Q_{s}$ and then it can be quantized and de-quantized without any loss at $Q_{s}$.

By decoding the bitstream $\operatorname{VLC}\left(E I_{R B, n}^{Q_{s}}\right)$ in the decoder, the identical reconstruction to $S P_{F B, n}^{r}$ can be obtained as explained below. First, the transformed and quantized coefficients of different predictions will not introduce any mismatch, that is, the value at position $\mathrm{C}$ in the encoder and at position D in the decoder (Fig. 5(c)) are exactly the same. In other words, both positions $\mathrm{C}$ and $\mathrm{D}$ can create the prediction $Q_{s}\left(T\left(\operatorname{Intrapred}\left(S I_{R B, n}^{r}\right.\right.\right.$, Intra Mode $\left.\left.\left._{R B, n}\right)\right)\right)$. Second, this prediction in the decoder adds the entropy decoded $\operatorname{VLC}\left(E I_{R B, n}^{Q_{s}}\right)$ to generate the reconstructed SIframe $S I_{R B, n}^{r}$, which is given by

$S I_{R B, n}^{r}=T^{-1}\left(Q_{s}^{-1}\left(E I_{R B, n}^{Q_{s}}+Q_{s}\left(T\left(\operatorname{Intrapred}\left(S I_{R B, n}^{r}\right.\right.\right.\right.\right.$, Intra_Mode $\left.\left.\left.\left.\left.e_{R B, n}\right)\right)\right)\right)\right)$ 
By putting (7) into (8), we obtain

$S I_{R B, n}^{r}=T^{-1}\left(Q_{s}^{-1}\left(S P_{F B, n}^{Q_{s}}\right)\right)$

Equations 5 and 6 indicate that $S P_{F B, n}^{Q_{s}}$ is synchronized at $Q_{s}$, Eq. 9 can be written as

$S I_{R B, n}^{r}=S P_{F B, n}^{r}$

Now $S I_{R B, n}^{r}$ of the RB is used to replace $S P_{F B, n}^{r}$ of the FB, which is needed during VCR operations. In this case, $S I_{R B, n}^{r}$ is a reference frame for decoding the next P-frame, and such replacement will not introduced any mismatch error, as shown in Fig. 6(a).

Figure 6(b) shows the $\mathrm{SI}_{\mathrm{FB}} / \mathrm{SP}_{\mathrm{RB}}$ pair which is used for switching from the FB to the RB at frame $n$. In this case, the reconstructed frame of $S I_{F B, n}\left(S I_{F B, n}^{r}\right)$ in the FB is used to replace the reconstructed frame of $S P_{R B, n}\left(S P_{R B, n}^{r}\right)$ in the RB. To avoid the problem of reference mismatch, the replacement of $S P_{R B, n}^{r}$ with $S I_{F B, n}^{r}$ should not introduce any mismatch error.

For encoding $S I_{F B, n}$ of the $\mathrm{SI}_{\mathrm{FB}} / \mathrm{SP}_{\mathrm{RB}}$ pair, it is similar to encode $S P_{F B, n}$ and the only difference is that $S I_{F B, n}$ does not use any reference frame. The macroblocks in $S I_{F B, n}$ are coded by using intra prediction, which means that in the encoding diagram as shown in Fig. 7(a), the prediction is generated by intra prediction from neighboring blocks. The prediction error between $O_{F B, n}$ and its intra prediction in the quantized transform domain, $E I_{F B, n}^{Q_{p}}$, is

$E I_{F B, n}^{Q_{p}}=Q_{p}\left(T\left(e i_{F B, n}\right)\right)$

where $e i_{F B, n}=O_{F B, n}-$ Intrapred $\left(S I_{F B, n}^{r}\right.$, Intra_Mode $\left._{F B, n}\right)$ and Intra_Mode ${ }_{F B, n}$ is an intra-prediction modes of the current block. To achieve identical reconstruction, $S I_{F B, n}^{r}$ should be synchronized at the quantization level $Q_{s}$. To do so, the prediction error $e i_{F B, n}^{r}$ are firstly reconstructed and then added to the prediction again. It can be written as

$I_{F B, n}^{r}=e i_{F B, n}^{r}+\operatorname{Intrapred}\left(S I_{F B, n}^{r}\right.$, Intra_Mode $\left._{F B, n}\right)$

where $e i_{F B, n}^{r}=T^{-1}\left(Q_{p}^{-1}\left(E I_{F B, n}^{Q_{p}}\right)\right)$. The reconstructed frame $I_{F B, n}^{r}$ is then transformed and quantized with $Q_{s}$. The quantized transform coefficients of $S I_{F B, n}^{r}$ and its reconstructed frame $S I_{F B, n}^{r}$ are

$S I_{F B, n}^{Q_{s}}=Q_{s}\left(T\left(I_{F B, n}^{r}\right)\right)$

and

$S I_{F B, n}^{r}=T^{-1}\left(Q_{s}^{-1}\left(S I_{F B, n}^{Q_{s}}\right)\right)$

respectively.

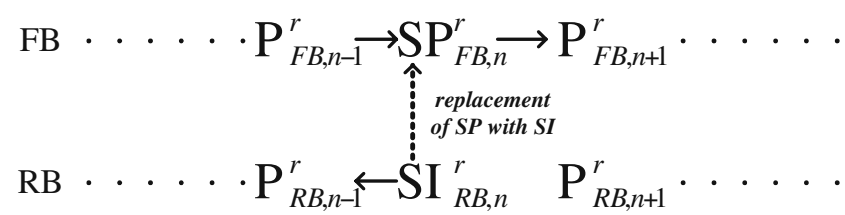

a

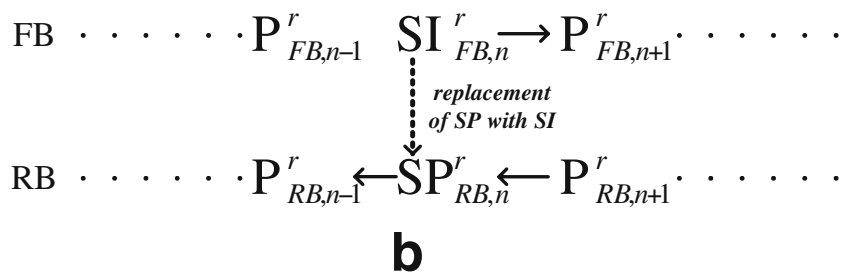

Figure 6 Frame replacement at (a) the $\mathrm{SP}_{\mathrm{FB}} / \mathrm{SI}_{\mathrm{RB}}$ pair and (b) the $\mathrm{SI}_{\mathrm{FB}} / \mathrm{SP}_{\mathrm{RB}}$ pair.

The lower part of Fig. 7(a) shows how to encode $S P_{R B, n}$ in the RB. The reference for which it uses is the reconstructed frame at frame $n+1\left(P_{R B, n+1}^{r}\right)$ in the RB and $S I_{F B, n}^{Q_{s}}$ acts as an input. Hence, the prediction error of $S P_{R B, n}$ in the quantized transform domain, $E_{R B, n}^{Q_{s}}$, is

$$
E_{R B, n}^{Q_{s}}=S I_{F B, n}^{Q_{s}}-Q_{s}\left(T\left(\operatorname{Interpred}\left(P_{R B, n+1}^{r}, m v_{R B, n}\right)\right)\right)
$$

where $m v_{R B, n}$ is the motion vectors of frame $n$ by using frame $n+1$ of the RB as the reference. This $E_{R B, n}^{Q_{s}}$ is then entropy encoded as $\operatorname{VLC}\left(E_{R B, n}^{Q_{s}}\right)$. The decoding processes of $\mathrm{SI}_{\mathrm{FB}} / \mathrm{SP}_{\mathrm{RB}}$ pair are depicted in Fig. 7(b) and (c) which are quite similar to that of $\mathrm{SP}_{\mathrm{FB}} / \mathrm{SI}_{\mathrm{RB}}$ pair. When the replacement of $S I_{F B, n}^{r}$ in the FB for $S P_{R B, n}^{r}$ in the RB is needed, the bitstream $\operatorname{VLC}\left(E_{R B, n}^{Q_{s}}\right)$ is decoded according to the block diagram in Fig. 7(c). Then $S P_{R B, n}^{r}$ is reconstructed as

$S P_{R B, n}^{r}=T^{-1}\left(Q_{s}^{-1}\left(E_{R B, n}^{Q_{s}}+Q_{s}\left(T\left(\operatorname{Interpred}\left(P_{R B, n+1}^{r}, m v_{R B, n}\right)\right)\right)\right)\right)$

By using Eqs. 14 and 15, Eq. 16 can be further simplified as

$S P_{R B, n}^{r}=S I_{F B, n}^{r}$

This indicates that $S I_{F B, n}^{r}$ can exactly replace $S P_{R B, n}^{r}$ without any mismatch when switching from the FB to the $\mathrm{RB}$ is needed. 
Figure 7 Encoding and decoding of an $\mathrm{SI}_{\mathrm{FB}} / \mathrm{SP}_{\mathrm{RB}}$.

(a) $\mathrm{SI}_{\mathrm{FB}}$ and $\mathrm{SP}_{\mathrm{RB}}$ encoding,

(b) $\mathrm{SI}_{\mathrm{FB}}$ decoding, and (c) $\mathrm{SP}_{\mathrm{RB}}$ decoding.

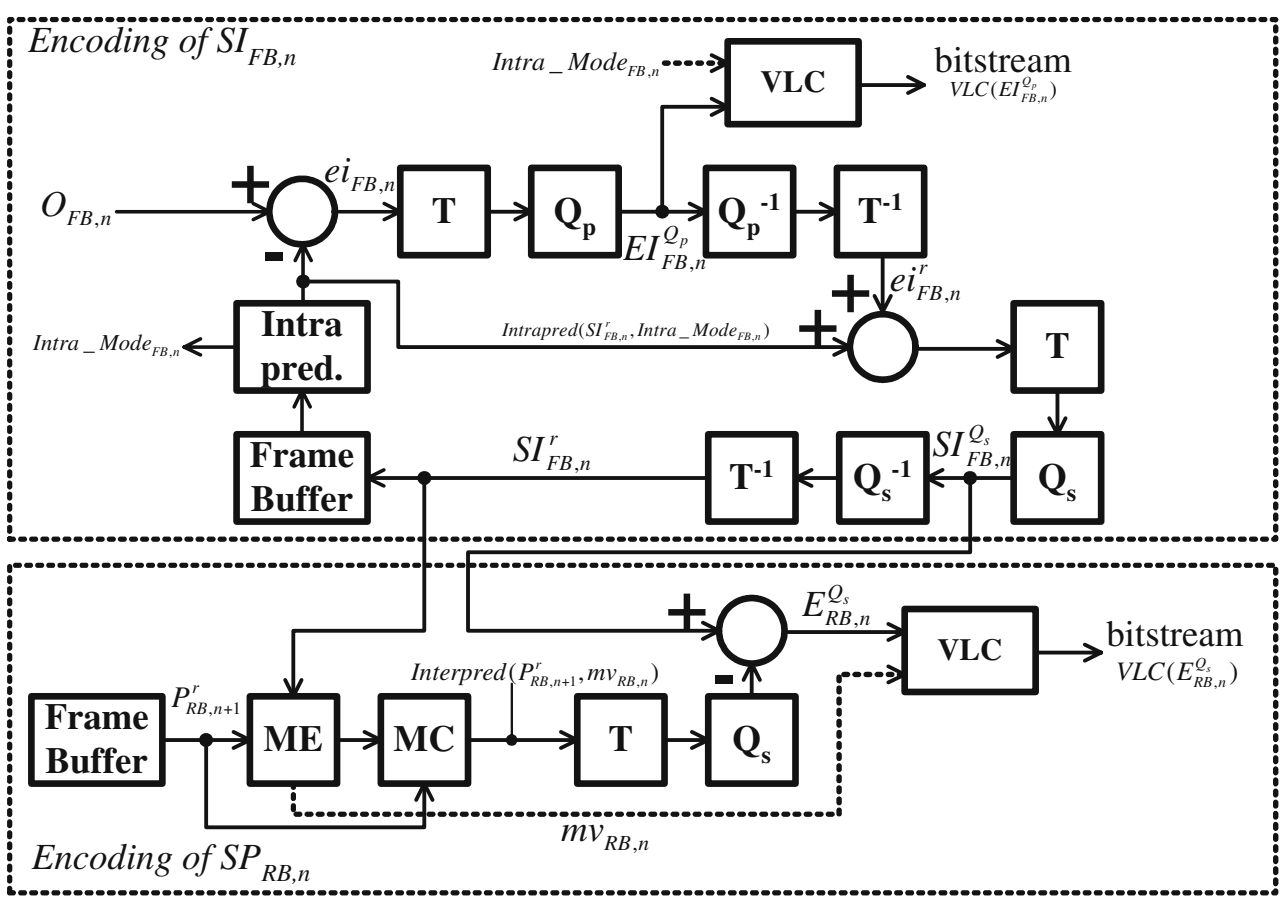

a

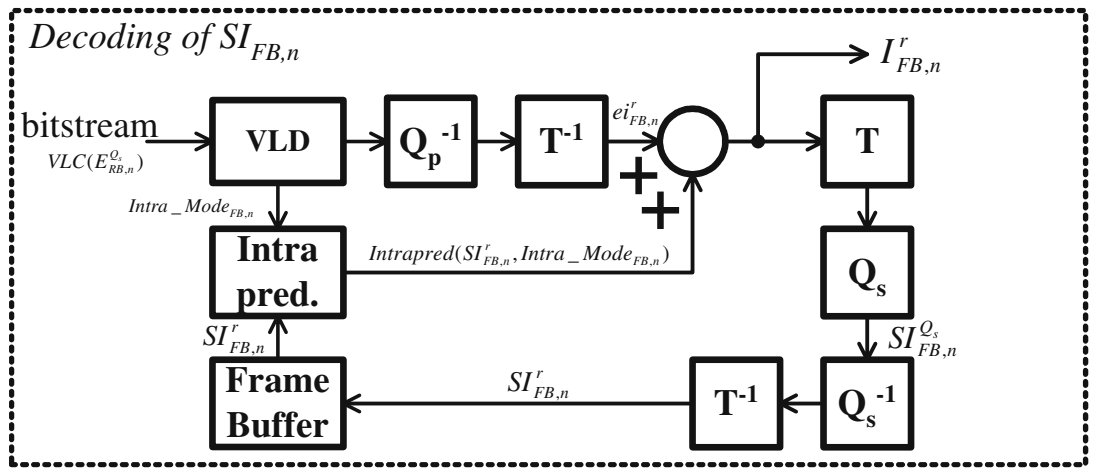

b

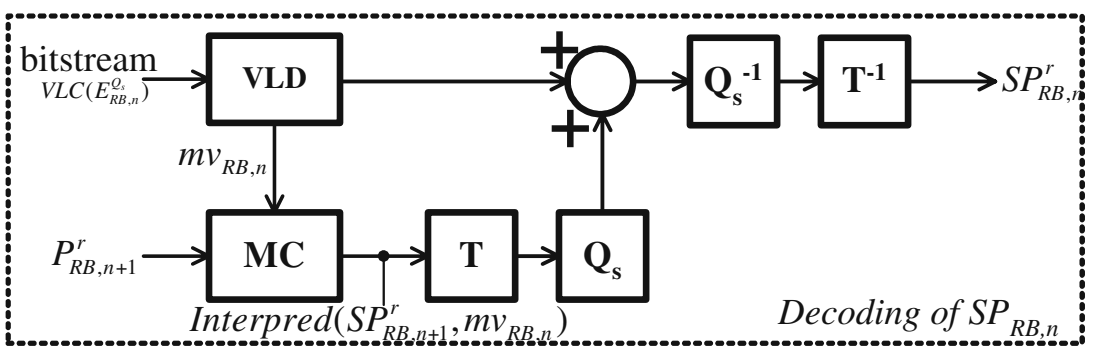

C

\section{Experimental Results}

In this section, we present some experimental results to evaluate the performance of the proposed dual-bitstream system. The H.264/AVC reference software JM [27] was employed to encode various video sequences with different spatial resolutions and motion characteristics. All test sequences have a length of 200 frames. "Carphone" and "Claire" are typical videophone sequences in QCIF (176× 144 pixels) format, while "Foreman", "Football" and "Table Tennis" are in either CIF $(352 \times 288$ pixels $)$ format or SIF $(352 \times 240$ pixels $)$ format. For the original dualbitstream system, each test sequence has been encoded into two bitstreams, FB and RB, and I-frames in the RB are 
Table 1 Average PSNR and bitrate comparison for original and proposed dual-bitstream systems.

\begin{tabular}{|c|c|c|c|c|c|c|c|}
\hline \multirow[t]{2}{*}{ Sequences } & \multirow[t]{2}{*}{ Streams } & \multicolumn{2}{|l|}{ Original } & \multicolumn{2}{|l|}{ Proposed } & \multirow[t]{2}{*}{$\Delta$ PSNR $(\mathrm{dB})$} & \multirow{2}{*}{$\begin{array}{l}\text { Percentage increase } \\
\text { in bitrate of the } \\
\text { proposed system }\end{array}$} \\
\hline & & PSNR (dB) & Bitrate (kbits/s) & PSNR (dB) & Bitrate (kbits/s) & & \\
\hline \multirow[t]{2}{*}{ Foreman $(352 \times 288)$} & FB & 35.838 & 331.933 & 35.820 & 348.787 & -0.017 & $5.08 \%$ \\
\hline & $\mathrm{RB}$ & 35.796 & 336.050 & 35.775 & 364.472 & -0.021 & $8.46 \%$ \\
\hline \multirow[t]{2}{*}{ Football $(352 \times 240)$} & FB & 33.461 & 946.258 & 33.459 & 961.187 & -0.002 & $1.58 \%$ \\
\hline & $\mathrm{RB}$ & 33.432 & 938.954 & 33.433 & 967.981 & 0.001 & $3.09 \%$ \\
\hline \multirow[t]{2}{*}{ Table Tennis $(352 \times 240)$} & FB & 32.824 & 528.680 & 32.805 & 545.380 & -0.020 & $3.16 \%$ \\
\hline & $\mathrm{RB}$ & 32.644 & 530.878 & 32.709 & 564.649 & 0.065 & $6.36 \%$ \\
\hline \multirow[t]{2}{*}{ Claire $(176 \times 144)$} & FB & 38.419 & 31.249 & 38.410 & 32.767 & -0.009 & $4.86 \%$ \\
\hline & $\mathrm{RB}$ & 38.423 & 30.443 & 38.402 & 34.144 & -0.022 & $12.16 \%$ \\
\hline \multirow[t]{2}{*}{ Carphone $(176 \times 144)$} & FB & 35.537 & 93.846 & 35.524 & 97.141 & -0.013 & $3.51 \%$ \\
\hline & $\mathrm{RB}$ & 35.534 & 93.469 & 35.500 & 103.368 & -0.034 & $10.59 \%$ \\
\hline
\end{tabular}

interleaved between I-frames in the FB. Note that a total of 52 values of the quantization level are supported by the H.264 standard, indexed by a Quantization Parameter (QP) which is in the range of $0-51$ and the quantization level doubles in size for every increment of 6 in QP. The wide range of the quantization levels makes it possible for an encoder to control the tradeoff accurately and flexibly between bit rate and quality. To encode the FB and the RB of the conventional system, QP was set to 30 for encoding $\mathrm{I} / \mathrm{P}$-frames. For all test sequences, the frame-rate of the video stream was 30 frames/s and the GOP length was fixed to 60 with an I-P structure.

Table 1 shows the PSNR and the bit rates of the FB and the $\mathrm{RB}$ for different sequences when the original system is used. In order to have a fair comparison between the original and proposed systems, we encoded the $\mathrm{SP}_{\mathrm{FB}} / \mathrm{SI}_{\mathrm{RB}}$ and $\mathrm{SI}_{\mathrm{FB}} / \mathrm{SP}_{\mathrm{RB}}$ pairs such that the reconstructed quality of the new dual bitstreams is similar to that of the original one. We also include in Table 1 the PSNR and the bit rates of different sequences when the new dual bitstreams are encoded using the proposed system. It can be observed in this table that the bitstream using SP/SI-frames have lower coding efficiency than the bitstream using P/I-frames. As a result, our system will introduce overhead in the bit rate, but it is not significant, especially in the normal playback. In this situation, only the FB is used and the increase in bit rate is fewer than $5.1 \%$. Note, however, that the proposed dual-bitstream system provides better reconstruction quality for various VCR operations as discussed in the following.

Besides, we have also created the drift-compensated bitstreams [15] for the original dual-bitstream system. In [15], the drift compensation (DC) needs only to store the drift-compensated frames for all the I-frames of the dual bitstreams in order to reduce the storage requirement of the server. As mentioned before, since the coding efficiency of $\mathrm{SP} / \mathrm{SI}$-frames has lower than that of P/I-frames, our system therefore introduces increase in the storage requirement ( $\Delta$ bits), as shown in Table 2. But it is still smaller than that of the original system with DC.

Table 3 then gives our experimental results on the average PSNR for different systems on all possible switching points between the FB and the RB. It is clear that the original system introduces quality degradation as compared with the quality of the original FB or RB. This is due to the fact that an I-frame of the FB (RB) is used to approximate a P-frame of the RB (FB) at the moment of fast forward/ reverse operations in the original system and this approximation leads to reference mismatch which affects the reconstructed quality of the next requested frame. However, the switching points of the proposed dual-bitstream system are at $\mathrm{SI}_{\mathrm{FB}} / \mathrm{SP}_{\mathrm{RB}}$ and $\mathrm{SP}_{\mathrm{FB}} / \mathrm{SI}_{\mathrm{RB}}$ pairs. These pairs can ensure identical frame reconstruction and no approximation is required. This means the reconstruction quality during the replacement of SP with SI is the same as that of the FB or RB. The advantage of the proposed system is indicated in the experimental results as shown in Table 3. This table shows that our proposed system has significant improvement over the original systems with and without DC for all video sequences.

Furthermore, for the original systems, the quality degradation will not only be confined to the frame at the

Table 2 Increases in the storage requirements of the original system with DC and the proposed system as compared to the original system.

\begin{tabular}{lcc}
\hline Sequences & Original with DC & Proposed \\
\hline Foreman & $8.41 \%$ & $6.78 \%$ \\
Football & $2.62 \%$ & $2.33 \%$ \\
Table Tennis & $5.92 \%$ & $4.76 \%$ \\
Claire & $9.35 \%$ & $8.46 \%$ \\
Carphone & $8.74 \%$ & $7.04 \%$ \\
\hline
\end{tabular}


Table 3 Average PSNR performance for conventional and proposed dual-bitstream systems on every possible switching between the FB and RB.

\begin{tabular}{|c|c|c|c|c|c|c|}
\hline \multirow[t]{2}{*}{ Sequences } & \multicolumn{2}{|l|}{ Original } & \multicolumn{2}{|c|}{$\begin{array}{l}\text { Original with DC (PSNR gain over original } \\
\text { without DC) }\end{array}$} & \multicolumn{2}{|c|}{$\begin{array}{l}\text { Proposed (PSNR gain over original } \\
\text { without DC) }\end{array}$} \\
\hline & $\mathrm{FB} \rightarrow \mathrm{RB}$ & $\mathrm{RB} \rightarrow \mathrm{FB}$ & $\mathrm{FB} \rightarrow \mathrm{RB}$ & $\mathrm{RB} \rightarrow \mathrm{FB}$ & $\mathrm{FB} \rightarrow \mathrm{RB}$ & $\mathrm{RB} \rightarrow \mathrm{FB}$ \\
\hline Foreman & 34.475 & 34.686 & $35.469(0.994)$ & $35.587(0.901)$ & $35.733(1.258)$ & $35.784(1.098)$ \\
\hline Football & 33.107 & 33.212 & $33.372(0.265)$ & $33.649(0.437)$ & $33.850(0.743)$ & $33.916(0.704)$ \\
\hline Table Tennis & 32.176 & 32.335 & $32.524(0.348)$ & $32.816(0.481)$ & $32.894(0.718)$ & $32.988(0.653)$ \\
\hline Claire & 37.516 & 37.324 & $37.985(0.469)$ & $38.269(0.945)$ & $38.175(0.659)$ & $38.587(1.264)$ \\
\hline Carphone & 34.218 & 34.583 & $35.112(0.894)$ & $35.500(0.917)$ & $35.536(1.318)$ & $35.642(1.059)$ \\
\hline
\end{tabular}

switching point but can propagate and be accumulated in the subsequent P-frames. Figure 8 shows the amount of drift in PSNR using the various systems for the "Football" sequence in the situation where the fast-reverse mode is initially operated and subsequently a normal-play request is launched. Assume that the user requests a normal-play request at the start of each GOP in the RB. In this case, it has the longest drift propagation. This figure shows that drift caused by the I-to-P approximation of the original system is very serious and lasts until the next I-frame in the FB. Although the system with DC can reduce drift propagation, it still happens. As expected, our proposed system does not introduce any drift and it follows the same PSNR of the FB and the RB. This result indicates that the visual quality of the reconstructed frames in the proposed system for various VCR operations is exactly identical to that of the normal playback.

\section{Conclusions}

In this paper, we propose to adopt the concept of SP/SIframe coding to improve the performance of the dualbitstream system which has been proven to be an efficient approach for providing VCR functionality in video streaming. The original dual-bitstream system stores a forwardencoded bitstream (FB) and a reverse-encoded bitstream (RB) in the server in order to reduce the requirement of network bandwidth significantly. However, a mismatch between encoding the FB and RB incurs. This induces the problem of drift when the I-to-P approximation is used for fast-forward and fast-reverse playbacks. Therefore, our novel dual-bitstream system is aimed at avoiding the problem of reference mismatch when a frame in the $R B$ (FB) is selected as a reference for a frame in the FB (RB). The proposed system modifies the dual bitstreams using the main feature of SP/SI-frames which can provide an identical reconstruction even when different reference frames are used for prediction. To utilize this property, $\mathrm{SP}_{\mathrm{FB}} / \mathrm{SI}_{\mathrm{RB}}$ and $\mathrm{SI}_{\mathrm{FB}} / \mathrm{SP}_{\mathrm{RB}}$ pairs are added in the structure of dual bitstreams and this arrangement ensures that the I-to-P approximation will not happen in our system for various VCR operations. Consequently, the new dual-bitstream system will not cause any mismatch at the decoder side and the quality of reconstructed frames can be maintained. Besides, we have also shown how $\mathrm{SP}_{\mathrm{FB}} / \mathrm{SI}_{\mathrm{RB}}$ and $\mathrm{SI}_{\mathrm{FB}} /$ $\mathrm{SP}_{\mathrm{RB}}$ pairs can be encoded such that identical frames can be reconstructed even when an SI-frame of one bitstream is used to replace an SP-frame of the other bitstream in any VCR operation. Experimental results show that the visual quality of all reconstructed frames in our new system for various VCR operations will be exactly the same as that of normal playback. Therefore, the proposed dual-bitstream system with SP/SI-frames is a promising solution for H.264 video streaming with VCR functionality.
Figure 8 Drift in PSNR of difference systems for the "Football" sequence. Negative value means PSNR drop of the reconstructed frame for the VCR operation as compared with that for the normal playback.

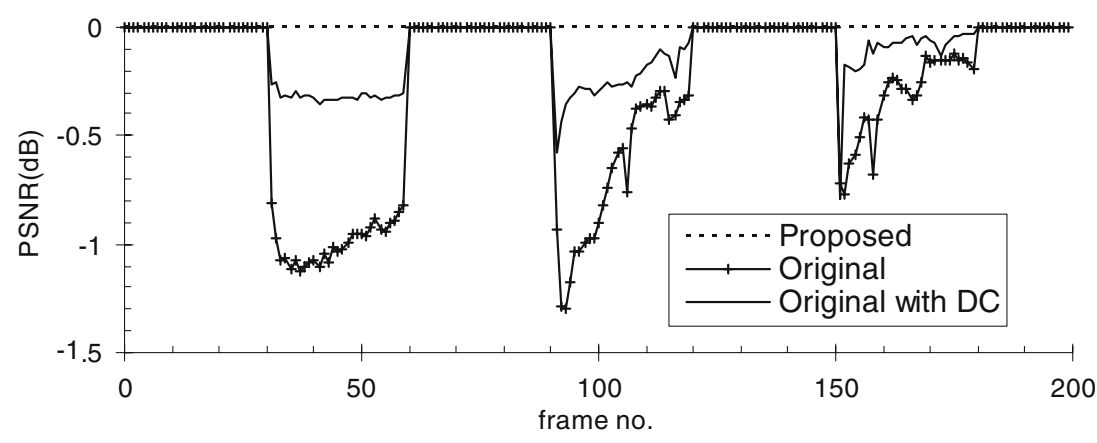


Acknowledgements The work described in this paper is partially supported by the Centre for Signal Processing, Department of Electronic and Information Engineering, Hong Kong Polytechnic University and a grant from the Research Grants Council of the Hong Kong Special Administrative Region, China (PolyU 5120/07E). Li-Kit Lai acknowledges the research studentships provided by the University.

\section{References}

1. Stockhammer, T., Jenkac, H., \& Kuhn, G. (2004). Streaming video over variable bit-rate wireless channels. IEEE Transactions on Multimedia, 6(2), 268-277.

2. Ganjam, A., \& Zhang, H. (2005). Internet multicast video delivery. Proceedings of the IEEE, 93(1), 159-170.

3. Real Networks RealPlayer [Online]. http://www.real.com/. Accessed 24 December 2009.

4. Microsoft Window Media, Microsoft Corporation Inc. [Online]. http://www.microsoft.com/windows/windowsmedia/. Accessed 24 December 2009.

5. SonicBlue Inc. [Online]. http://www.replay.com/. Accessed 24 December 2009.

6. TiVo Inc. [Online]. http://www.tivo.com/. Accessed 24 December 2009.

7. ISO/IEC 11172-2: 'Information Technology_Coding of Moving Pictures and Associated Audio for Digital Storage Media at up to about 1.5 Mbit/s - Part 2: Video', 1993.

8. ISO/IEC 13818-2: 'Information Technology-Generic Coding of Moving Pictures and Associated Audio Information: Video', 1996.

9. ISO/IEC 14996-2: 'Coding of Audio-visual Objects-Part 2: Visual', 2001.

10. ITU-T Rec. H.263: 'Video Coding for Low Bitrate Communication', 1997.

11. ISO/IEC 14996-10 and ITU-T Rec. H.264: 'Advanced Video Coding', 2003.

12. Srinivasan, R., \& Rao, K. R. (1985). Predictive coding based on efficient motion estimation. IEEE Transactions on Communication, 33(9), 1011-1015.

13. Hui, K. C., \& Siu, W. C. (2007). Extended analysis of motioncompensated frame difference for block-based motion prediction error. IEEE Transactions on Image Processing, 16(5), 1232-1245.

14. Chan, Y. L., \& Siu, W. C. (2001). An efficient search strategy for block motion estimation using image features. IEEE Transactions on Image Processing, 10(8), 1223-1238.

15. Lin, C. W., Zhou, J., Youn, J., \& Sun, M. T. (2001). MPEG video streaming with VCR functionality. IEEE Transactions on Circuits and Systems for Video Technology, 11(3), 415-425.

16. Huang, S. Y. (2003). Improved techniques for dual-bitstream MPEG video streaming with VCR functionalities. IEEE Transactions on Consumer Electronics, 49(4), 1153-1160.

17. Ip, T. P., Chan, Y. L., \& Siu, W. C. (2008). Redundancy reduction technique for dual-bitstream MPEG video streaming with VCR functionalities. IEEE Transactions on Broadcasting, 54(3), 412-418.

18. Fu, C. H., Chan, Y. L., \& Siu, W. C. (2006). Efficient reverse-play algorithms for MPEG video streaming with VCR support. IEEE Transactions on Circuits and Systems for Video Technology, 16(1), 19-30.

19. McManus, J. M., \& Ross, K. W. (1996). Video-on-demand over ATM: constant-rate transmission and transport'. IEEE Journal on Selected Areas in Communications., 14(6), 1087-1098.

20. Fung, K. T., Chan, Y. L., \& Siu, W. C. (2002). New architecture for dynamic frame-skipping transcoder. IEEE Transactions on Image Processing, 11(8), 886-900.
21. Tan, Y. P., Liang, Y., \& Yu, J. (2002). Video transcoding for fast forward/reverse video playback. Proceedings of International Conference on Image Processing, 713-716.

22. Omoigui, N., He, L., Gupta, A., Grudin, J., \& Sanocki, E. (1999). Time-compression: system concerns, usage, and the benefits. Proceedings of ACM SIGHI Conference, 136-143.

23. Karczewicz, M., \& Kurceren, R. (2003). The SP- and SI-Frames design for H.264/AVC. IEEE Transactions on Circuits and Systems for Video Technology, 13(7), 637-644.

24. Kurceren, R., \& Karczewicz, M. (2002). Synchronizationpredictive coding for video compression: the SP frames design for JVT/H.26L. Proceedings of IEEE International on Conference Image Processing, 2002, 497-500.

25. Lai, K. K., Chan, Y. L., Fu, C. H., \& Siu, W. C. (2008). Viewpoint switching in multiview videos using SP-frames. Proceedings of the IEEE International Conference on Image Processing, 1776-1779.

26. Lai, K. K., Chan, Y. L., \& Siu, W. C. (2007). A quantized transform-domain motion estimation technique for H.264 secondary SP-Frames. Proceedings of the 8th Pacific Rim Conference on Multimedia, 128-137.

27. JVT Reference Software JM15.1 [Online] http://iphome.hhi.de/ suehring/tml/download/. Accessed 24 December 2009.

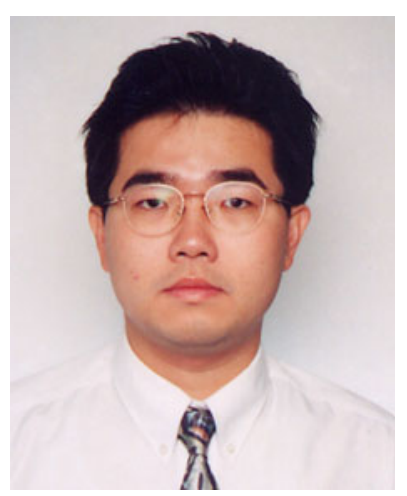

Yui-Lam Chan received the BEng with a First Class Honours degree and the Ph.D degree from The Hong Kong Polytechnic University in 1993 and 1997, respectively. During his studies, he was the recipients of more than 10 famous prizes, scholarships and fellowships for his outstanding academic achievement, such as being the Champion in Varsity Competition in Electronic Design, the Sir Edward Youde Memorial Fellowship, and the Croucher Foundation Scholarships. Dr Chan joined The Hong Kong Polytechnic University in 1997, and is now an Assistant Professor in the Department of Electronic and Information Engineering. He received both of the Faculty Merit Award in Teaching (Team) and the Faculty of Engineering Research Grant Achievement Award in 2005. Dr Chan is also actively involved in professional activities. In particular, he serves as a reviewer and Session Chairman for many international journals/conferences. He was the Registration Chair of the 2004 International Symposium on Intelligent Multimedia, Video and Speech Processing (ISIMP' 2004) held in Hong Kong between October 20 and 22, 2004. Besides, he is the Secretary of the coming 2010 IEEE International Conference on Image Processing (ICIP'2010). He has published over 60 research papers in various international journals and conferences. His research and technical interests include multimedia technologies, signal processing, image and video compression, video streaming, video transcoding, video conferencing, digital TV/HDTV, 3DTV, multi-view video coding, future video coding standards, error-resilient coding, and digital VCR. 


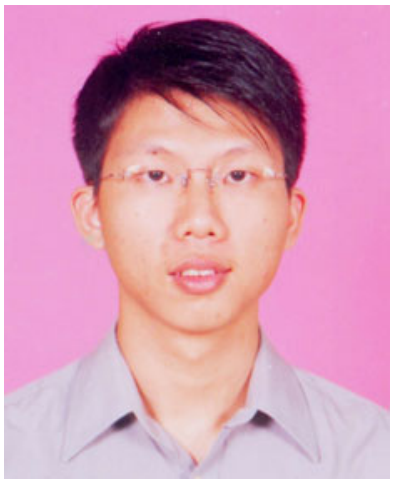

Tak-Piu Ip received the BSc(Hons) degree and Ph.D degree from The Hong Kong Polytechnic University in 2003 and 2007, respectively. His research interests include image and video technology, video compression and digital VCR.

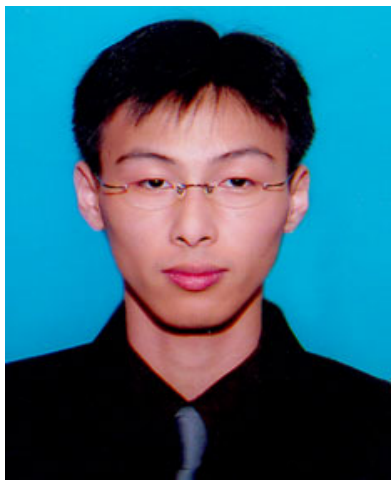

Ki-Kit Lai received the MEng degree and BEng(Hons) degree in Electronic and Information Engineering from The Hong Kong Polytechnic University in 2006. He is currently a Ph.D student at the same University under the supervision of Dr Y.L. Chan and Prof. W.C. Siu. His research interests include image and video technology, video compression and multi-view video coding.

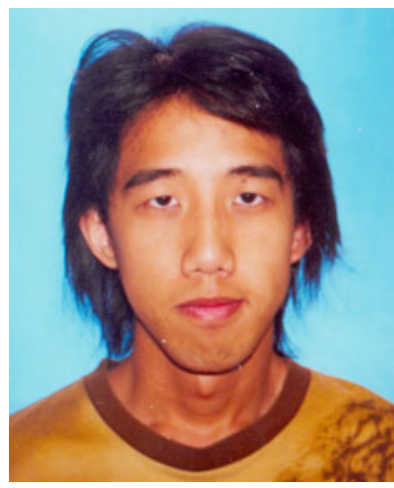

Chang-Hong Fu received the BEng with a First Class Honours degree and the Ph.D degree from The Hong Kong Polytechnic
University in 2002 and 2008, respectively. He is currently a Postdoctoral Fellow at The Hong Kong Polytechnic University. His research interests include multimedia technologies, signal processing, digital VCR, multi-view video coding and 3DTV.

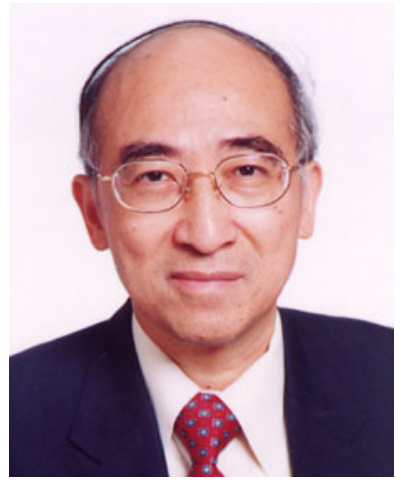

Wan-Chi Siu received the Associateship from The Hong Kong Polytechnic University and the MPhil degree from The Chinese University of Hong Kong in 1975 and 1977, respectively, and the Ph. D. Degree from Imperial College of Science, Technology \& Medicine, London, UK, in October 1984. He was with The Chinese University of Hong Kong as a Tutor and later as an Engineer between 1975 and 1980. He then joined The Hong Kong Polytechnic University as a Lecturer in 1980. He was promoted to Senior Lecturer, Principle Lecturer, and Reader in 1985, 1987 and 1990, respectively, and has been Chair Professor of the Department of Electronic and Information Engineering since 1992. Professor Siu is now Director of the Centre for Multimedia Signal Processing of the same university. $\mathrm{He}$ is an expert, and a popular speaker in Digital Signal Processing, specializing in fast algorithms and video coding. His research interests also include transforms, image processing, and the computational aspects of pattern recognition and wavelets. He frequently makes open presentations and has published 380 research papers, over 150 of which appeared in international journals, such as IEEE Transactions. His work on fast computational algorithms (such as DCT) and motion estimation algorithms have been well accepted by academic peers, with good citation records, and a number of which are now being used in hi-tech industrial applications. He is an editor of the book, 'Multimedia Information Retrieval and Management', Springer Berlin Heidelberg 2003. Professor Siu has been/was Guest Editor, Associate Editor and Member of editorial board of a number of journals, including IEEE Transactions on Circuits and Systems, Pt. II, Pattern Recognition, Journal of VLSI Signal Processing Systems for Signal, Image, Video Technology, the EURASIP Journal on Applied Signal Processing, in addition to other journals. He has been a keynote/invited speaker and key organizer of many worldclass international conferences (such as IEEE ICNNSP'08 (keynote speaker, China), IEEE ICICS'07 (invited speaker, Singapore), IEEE CPM'2002 (keynote speaker, Taiwan), IEEE MMSP'08 (General Co-Chair, Australia) and some IEEE society sponsored flagship conferences: ISCAS'97 (technical program chair), ICASSP'2003 (general chair) and ICIP'2010 (general chair)). In 1994 he chaired the first Engineering and Information Technology Panel of the Research Assessment Exercise (RAE) to assess the research quality of 19 Cost Centers (departments) from all universities in Hong Kong. 